\title{
NONEXISTENCE OF QUASI-INVARIANT MEASURES ON INFINITE-DIMENSIONAL LINEAR SPACES ${ }^{1}$
}

\author{
JACOB FELDMAN
}

It was shown by V. N. Sudakov [7] that a locally convex topological linear space with a nontrivial quasi-invariant $\sigma$-finite measure on its weakly measurable sets must be finite-dimensional. Sudakov's ingenious proof uses a theorem of Ulam [6, Footnote 3]. In some unpublished notes [8], Y. Umemara attempted to prove the same theorem, by utilizing Weil's "converse to the existence of Haar measure" [9, Appendix 1]. Umemara's proof was incorrect. However, the approach seemed a natural one. In the present article Weil's theorem will be utilized to prove a more general nonexistence theorem, from which Sudakov's theorem may immediately be deduced, but which also covers the case of a Borel measure on any metrizable linear space; for example, the equivalence classes of measurable functions on a measure space.

Thanks are due to R. Dudley and J. Mermin for patient and critical listening, and to C. C. Moore for contributing an essential step to an earlier version of the proof. $R$ will always denote the real line, and $T$ the unit circle in the complex plane.

THEOREM 1. Let $Y$ be a locally compact abelian group, which is also a real vector space. Assume that for each real a and each $\hat{y}$ in the character group $\hat{Y}$ of $Y$, the map $y \rightarrow \hat{y}(a y)$ is continuous. A ssume also that the map $a \rightarrow y(a y)$ is measurable, using Lebesgue measurable sets in $R$ and Baire sets in T. Then $Y$ is finite-dimensional.

Proof. $a \rightarrow \hat{y}(a y)$ is a homomorphism from $R$ under addition to $T$ under multiplication. The measurability assumption makes it continuous, by [4, Theorem 9.3.1]. Then, by [2, Corollary 2.1], $a \rightarrow a y$ is continuous. It follows that $Y$ is connected, since each $y$ may be connected to 0 by the curve $a y: 0 \leqq a \leqq 1$. We may define $a \hat{y}$ by the equation $(a \hat{y})(y)=\hat{y}(a y)$. Then $a \rightarrow a \hat{y}$ is likewise continuous, and so an analogous argument shows that $\hat{Y}$ is connected. But connectedness of $Y$ says there is an isomorphism $i=Y \rightarrow R^{n} \times C, C$ compact: see $\left[9\right.$, p. 110]. So $\hat{\imath}: \hat{Y} \leftarrow \hat{R}^{n} \times \hat{C}$. Now, $\hat{R}^{n}$ is just $R^{n}$, and $\hat{C}$ is discrete. The only way that $R^{n} \times \hat{C}$ can be connected, therefore, is for $\hat{C}$ to consist of one element, and consequently likewise for $C$. So $i: Y \rightarrow R^{n}$.

Received by the editors May 11, 1964 and, in revised form, June 5, 1964.

1 Written with partial support from NSF Contract GP-2. 
The map is automatically a linear space isomorphism over the $r a$ tionals, that is $i(r y)=r(i y)$ for rational $r \in R$. But then continuity implies $i(a y)=a i(y)$ for each $a \in R$.

Example 1. Let $R_{d}$ be the discrete reals. Their dual, $\hat{R}_{d}$, otherwise known as the Bohr group, is of course compact. $\hat{R}_{d}$ is a vector space, under the dual operation to the obvious scalar multiplication in $R_{d}$. $\hat{R}_{d}$ is an infinite-dimensional vector space, and also a compact abelian group. The scalar operations are homeomorphic automorphisms of $\hat{R}_{d}$. However, if $y \neq 0$ in $\hat{R}_{d}$, the map $a \rightarrow a y$ is not measurable, so this does not contradict Theorem 1 .

THEOREM 2. Let $X$ be a real linear space, and $\S$ a $\sigma$-ring of subsets. Assume

(1) $x \rightarrow a x$ is measurable for each $a \in R$.

(2) $a \rightarrow a x$ is measurable for each $x \in X$ (with respect to Lebesgue measurable sets in $R$ ).

(3) $(x, y) \rightarrow x+y$ is measurable, using the ordinary product $\sigma$-ring in $X \times X$.

Note that (3) implies measurability of $x \rightarrow x+y$, for each $y \in X$. Assume also the existence of a nonzero $\sigma$-finite and separable measure $\mu$ on $\delta$ such that

(4) $\mu(S)=0 \Rightarrow \mu(S+x)=0$, for each $S \in S$ and $x \in X$, i.e. $\mu$ is quasiinvariant.

(5) If $x \neq 0$ then $\exists S \in S$ such that the symmetric difference of $S$ and $S+x$ has positive $\mu$-measure.

Conclusion. $X$ is finite-dimensional.

Proof. First: there is actually a translation-invariant $\sigma$-finite measure on $S$, equivalent to $\mu$. This is not difficult to see directly, but is also a special case of [5, Lemma 7.3]. Furthermore, the invariant measure is unique, up to a constant multiple. This can be seen as follows.

Let $\alpha, \beta$ be $\sigma$-finite invariant measures on $\mathcal{s}$. We may assume $\alpha \prec \beta$. Then $f=d \alpha / d \beta$ is translation-invariant, up to $\beta$-null sets. We show $f$ is constant $\beta$-a.e. For let $S=\{x: f(x)<a\}$.

Let $d \gamma=1 s^{\perp} d \beta$. Then, since $S$ is invariant up to $\beta$-null sets, $\gamma$ is an invariant measure.

Now, $\gamma(S)=0$, so $\gamma(S-x)=0$ for all $x$, and $0=\int \gamma(S-x) d \beta(x)$ $=\iint 1_{S}(y+x) d \gamma(y) d \beta(x)$. By Fubini, $1_{S}(y+x)$ is $0 \beta$-a.e., for $\gamma$-a.e. $y$. So either $\gamma$ is the zero measure (in which case $S^{\perp}$ is $\beta$-null), or else $\exists y$ such that $\beta(S-y)=0$, so $S$ is $\beta$-null. That is, either $\{x: f(x)<a\}$ or $\{x: f(x) \geqq a\}$ is null.

Hereafter, then, we denote by $\rho$ this unique (up to constant multi- 
ple) $\sigma$-finite invariant measure on $\delta$. Weil's theorem, which we utilize in the form given in $[3, \S 62]$, tells us that there is a canonical uniformity $u$ on $X$ such that the completion $Y$ of $X$ in this uniformity is a locally compact group. Denoting by $B$ the Baire sets of $Y$, we also have $\delta \supset \otimes \mid X$, and $\sigma(B)=\rho(B \cap X)$ is a Haar measure on $B$. $X$ being abelian, $Y$ is likewise.

Let $\phi_{a}$ be the map $x \rightarrow a x$ from $X$ to $X$. Then $\rho \circ \phi_{a}^{-1}$ is easily seen to be again an invariant measure, hence is a constant multiple of $\rho$. So the uniformity induced by $\rho \circ \phi_{a}^{-1}$ is the same as that induced by $\rho$, and consequently $\phi_{a}$ extends to a homeomorphic automorphism $\psi_{a}$ of $Y$.

Separability of $\mu$ implies separability of $\rho$, which in turn implies separability of $\sigma$. I claim this implies that $Y$ has a countable complete neighborhood base at 0 . For let $B_{1}, B_{2}, \cdots$ be a sequence of sets of finite measure in $B$ which generate $B$ up to $\sigma$-null sets. Then an examination of the definition of the topology in $Y$ shows that

$$
\left\{\left\{x: \sigma\left(\left(B_{n}+x\right) \Delta B_{n}\right)<\frac{1}{m}\right\}: n, m=1,2, \cdots\right\}
$$

is a complete system of open neighborhoods of zero in $Y$.

Thus, for each $y \in Y$ there is a sequence $x_{1}, x_{2}, \cdots$ in $X$ with $x_{n} \rightarrow y$ (since $X$ is dense in $Y$ ). Consequently, if $\hat{y} \in \hat{Y}, \hat{y}\left(\psi_{a}(y)\right.$ ) $=\hat{y}\left(\psi_{a}\left(\lim _{n \rightarrow \infty} x_{n}\right)\right)=\hat{y}\left(\lim _{n \rightarrow \infty} \psi_{a}\left(x_{n}\right)\right)=\lim _{n \rightarrow \infty} \hat{y}\left(a x_{n}\right)$.

Now, the embedding from $X$ into $Y$ is measurable from $S$ into $B$; consequently, $a \rightarrow \hat{y}\left(a x_{n}\right)$ is measurable from the Lebesgue-measurable sets in $R$ to the Baire sets in $T$. Thus, the same holds for their pointwise limit $a \rightarrow \hat{y}\left(\psi_{a}(y)\right)$. The map $a \rightarrow \psi_{a}(y)$ is now easily seen to make $Y$ into a vector space satisfying the assumptions of Theorem 1 , so $Y$ is finite-dimensional, and so is its vector subspace $X$.

Corollary. Let $X$ be a metrizable topological linear space. Let there be a nontrivial $\sigma$-finite quasi-invariant measure $\nu$ on the Borel sets of $X$. Then $X$ is finite-dimensional.

Proof. We may assume that $\nu$ has total mass 1 . Notice that if $S$ is any open neighborhood of zero, then $\bigcup_{n=1}^{\infty} n S=X$, so $\nu(n S)>0$ for some $n$. Consequently, $\sum_{n=1}^{\infty}\left(1 / 2^{n}\right) \nu(n S)>0$. Now define a Borel measure $\nu_{n}$ by $\nu_{n}(B)=\nu(n B)$. This makes sense, since $n B$ is Borel for any Borel set $B$, because of continuity of the map $x \rightarrow(1 / n) x$. Furthermore, $\nu_{n}$ is quasi-invariant. Define $\mu(B)=\sum_{n=1}^{\infty}\left(1 / 2^{n}\right) \nu_{n}(B)$. Then $\mu$ is again a quasi-invariant Borel measure, $\nu(X)=1$, and $\mu(S)$ $>0$ for any open neighborhood of zero. It will be shown that $X$, its 
Borel sets, and $\mu$, satisfy the condition of Theorem 2 .

(1), (2) and (4) are already clear. (5) may be seen as follows: if $x \neq 0$, then choose an open neighborhood $S$ of 0 with $x \in S-S$. Then $S$ is disjoint from $S+x$, so (5) is satisfied. It remains to show that $\mu$ is separable, and that $(x, y) \rightarrow x+y$ is product-measurable. These will both follow if it can be shown that $X$ is a separable metric space, which we do by exhibiting a countable dense subset.

Let $S_{n}(x)$ be the open $1 / n$-sphere about $x$, in some fixed metric. Then for some countable family $C_{n} \subset X, \bigcup_{x \in C_{n}} S_{n}(x)$ contains $X$ up to a set of measure 0 , since each $S_{n}(x)$ has positive measure. So, letting $C=\mathrm{U}_{n=1}^{\infty} C_{n}$, it follows that, for each $n, \bigcup_{x \in C} S_{n}(x)$ contains $X$ up to a set of measure zero.

I claim $C$ is dense. For if $y$ is not in the closure of $C$, then $\exists n$ such that $S_{n}(y) \cap C=\varnothing$, hence $S_{2 n}(y) \cap \bigcup_{x \in C} S_{2 n}(x)=\varnothing$. But $S_{2 n}(y)$ has positive measure; contradiction.

REMARK. The corresponding result for locally convex topological linear spaces easily reduces to the metrizable case, in fact to the case where the dual space is countably-dimensioned.

Example 2. The results of Elliott and Morse in [1] make the following construction possible.

Let $X$ be a countable product of real lines, and $\delta$ the usual $\sigma$-ring generated by the cylinders over finite-dimensional Baire bases. Then there is a measure $\rho$ on $\delta$ such that if $A_{n}$ is a Baire set on the real line, and the products of the Lebesgue measures of the $A_{n}$ form an infinite product converging to the number $\alpha$, then $\left\{x: x_{1} \in A_{1}\right.$, $\left.x_{2} \in A_{2}, \cdots\right\}$ is given measure $\alpha$ by the measure $\rho \cdot \rho$ is an invariant measure, and all assumptions of Theorem 2 are satisfied, except those of separability and $\sigma$-finiteness of the measure. In fact, $X$ cannot be embedded as a thick subgroup of a locally compact topological group; for suppose it were. Let $E$ be $x: 0 \leqq x_{n} \leqq 1$ for all $\left.n\right\}$; then $\rho(E)=1$, and $N=\{x: \rho(E \Delta(E+x))<1 / 4\}$ is a neighborhood of zero in the induced topology, but $N \subset\left\{x:\left|x_{n}\right|<1 / 4\right.$ for all $\left.n\right\}$, so $N$ is a subset of a set of measure zero, contradicting the assumption that $X$ is thick. "Thick" is used here as in $[3$, p. 275].

\section{REFERENCES}

1, E. O. Elliott and A. P. Morse, General product measures, Trans. Amer. Math. Soc. 109 (1964), 245-283.

2. I. Glicksberg, Uniform boundedness for groups, Canad. J. Math. 14 (1962), 269-276.

3. P. R. Halmos, Measure theory, Van Nostrand, New York, 1950.

4. E. Hille and R. S. Phillips, Functional analysis and semi-groups, rev. ed., Amer. Math. Soc. Colloq. Publ. Vol. 31, Amer. Math. Soc., Providence, R. I., 1957. 
5. G. W. Mackey, Borel structure in groups and their duals, Trans. Amer. Math. Soc. 85 (1957), 134-165.

6. J. T. Oxtoby and S. M. Ulam, On the existence of a measure invariant under a transformation, Ann. of Math. 40 (1939), 560-566.

7. V. N. Sudakov, Linear sets with quasi-invariant measure, Dokl. Akad. Nauk SSSR 127 (1959), 524-525. (Russian)

8. Y. Umemara, Measures on infinite dimensional vector spaces, dittoed seminar notes from Kyoto University.

9. A. Weil, L'intégration dans les groupes topologiques et ses applications, Actualités Sci. Ind., No. 869, Hermann, Paris, 1940.

Berkeley, California

\title{
A NOTE ON THE RECURSIVE UNSOLVABILITY OF PRIMITIVE RECURSIVE ARITHMETIC
}

\author{
RICHARD E. GRANDY ${ }^{1}$
}

We wish to show the recursive unsolvability of primitive recursive arithmetic (PRA). By PRA we mean a quantifier-free formal system of arithmetic which has expressions for all primitive recursive functions. In such a system all valid variable free formulas are provable and both of the Gödel incompleteness theorems hold. Further, we may define in the system bounded quantifiers and (for a suitable Gödel numbering) the following primitive recursive functions: th $(x)$, a function which enumerates the Gödel numbers of theorems of PRA, and $\operatorname{sub}(n, m)$, the function whose value is the Gödel number of the formula obtained by replacing the first variable in alphabetic order by the numeral $n$ through the formula number $m .^{2}$

If there is a recursive decision procedure for PRA, then the set of Gödel numbers of nontheorems is recursively enumerable. But if a set is recursively enumerable then it is primitive recursively enumerable. Thus if PRA is solvable there is a primitive recursive function whose range is precisely the set of Gödel numbers of nontheorems.

Assume there exists such a function $f$. Consider the formula

$$
\operatorname{th}(x)=\operatorname{sub}\left(x_{0}, x_{0}\right) \supset(E z) . z \leqq x \& f(z)=\operatorname{sub}\left(x_{0}, x_{0}\right) .
$$

Received by the editors March 15, 1965.

1 The author is indebted to J. R. Guard for his encouragement and to the National Science Foundation for financial support.

2 Detailed proofs may be found in J. R. Guard, The independence of transfinite induction up to $\omega^{\omega}$ in recursive arithmetic, unpublished dissertation, Princeton University, 1962, or H. E. Rose, On the consistency and undecidability of recursive arithmetic, Z. Math. Logik Grundlagen Math. 7 (1961), 124-135. 